\title{
Multinorms and Approximate Amenability of Weighted Group Algebras
}

\author{
Saman Ghaderkhani \\ Department of Mathematical Sciences, Isfahan University of Technology, P.O. Box 84156-83111, Isfahan, Iran \\ Correspondence should be addressed to Saman Ghaderkhani; s.ghaderkhani@math.iut.ac.ir \\ Received 18 August 2013; Accepted 30 December 2013; Published 23 February 2014 \\ Academic Editors: H. Lin, H. You, and C.-J. Zhao \\ Copyright (C) 2014 Saman Ghaderkhani. This is an open access article distributed under the Creative Commons Attribution License, \\ which permits unrestricted use, distribution, and reproduction in any medium, provided the original work is properly cited. \\ Let $G$ be a locally compact group, and take $p, q$ with $1 \leq p, q<\infty$. We prove that, for any left $(p, q)$-multiinvariant functional \\ on $L^{\infty}(G)$ and for any weight function $\omega \geq 1$ on $G$, the approximate amenability of the Banach algebra $L^{1}(G, \omega)$ implies the left \\ $(p, q)$-amenability of $G$, but in general the opposite is not true. Our proof uses the notion of multinorms. We also investigate the \\ approximate amenability of $M(G, \omega)$.
}

Dedicated with respect to my mother and my father

\section{Introduction}

Ghahramani and Loy in [1] introduced the notion of approximately amenable Banach algebras and, among other interesting results, they proved that for a locally compact group $G$, the group algebra $L^{1}(G)$ is approximately amenable if and only if $G$ is amenable.

Let $G$ be a locally compact group. Recently Dales et al. in [2] defined another generalized notion of amenability, called left $(p, q)$-amenability of $G$, for any $p, q$ such that $1 \leq p \leq q<$ $\infty$. The aim of the present paper is to show that, for any left $(p, q)$-multiinvariant function on $L^{\infty}(G)$ and for any weight function $\omega \geq 1$ on $G$, the approximate amenability of the Banach algebra $L^{1}(G, \omega)$ implies the left $(p, q)$-amenability of $G$, but in general the opposite is not true.

\section{Notation and Preliminaries}

In this section, we will recall various notations that we will use and give the definitions and some conventions.

2.1. Weighted Group Algebras and Approximate Amenability of Banach Algebras. Let $G$ denote a locally compact group with a fixed left Haar measurable $\lambda$ and $\omega$ a weight function on $G$; that is, a Borel measurable function $\omega: G \rightarrow \mathbb{R}^{+}$such that

$$
\omega(x \cdot y) \leq \omega(x) \omega(y), \quad(x, y \in G) .
$$

The weighted group algebra $L^{1}(G, \omega)$ is the space of all measurable complex-valued functions $f$ on $G$ such that

$$
\int_{G}|f(x)| \omega(x) d x<\infty
$$

and is equipped with the convolution product $*$ of functions; that is, for $f, g \in L^{1}(G, \omega)$ and $x \in G$,

$$
(f * g)(x)=\int_{G} f\left(x y^{-1}\right) g(y) d y,
$$

and the norm

$$
\|f\|_{\omega}=\int_{G}|f(x)| \omega(x) d x .
$$

Also, let $L^{\infty}\left(G, \omega^{-1}\right)$ be the space of all measurable complexvalued functions $\phi$ on $G$, such that $\phi / \omega$ is essentially bounded, and for $\phi \in L^{\infty}\left(G, \omega^{-1}\right)$ define

$$
\|\phi\|_{\infty, \omega}=\left\|\frac{\phi}{\omega}\right\|_{\infty}=\operatorname{ess} \sup \left\{\left|\frac{\phi(x)}{\omega(x)}\right| \mid x \in G\right\} .
$$


The spaces $L^{1}(G, \omega)$ and $L^{\infty}\left(G, \omega^{-1}\right)$ are in duality by

$$
\langle f, \phi\rangle=\int_{G} f \phi d \lambda \quad\left(f \in L^{1}(G, \omega), \phi \in L^{\infty}\left(G, \omega^{-1}\right)\right),
$$

and if $\omega(x) \geq 1,(x \in G)$, then $L^{\infty}(G) \subseteq L^{\infty}\left(G, \omega^{-1}\right)$. Let $M(G, \omega)$ be the Banach space of all complex-valued, regular Borel measures $\mu$ on $G$ such that

$$
\|\mu\|_{\omega}=\int_{G} \omega(x) d|\mu|(x)<\infty .
$$

Note that $M(G, 1)=M(G)$. If $\omega(x) \geq 1,(x \in G)$, then $M(G, \omega)$ is a subalgebra of $M(G)$.

Let $\mathscr{A}$ be a Banach algebra and $X$ a Banach $\mathscr{A}$-bimodule. A bounded linear map $D: \mathscr{A} \rightarrow X$ is called a derivation if

$$
D(a b)=D(a) \cdot b+a \cdot D \cdot(b) \quad(a, b \in \mathscr{A}) .
$$

For every $x \in X$ we define $a d_{x}^{\mathscr{A}}$ by

$$
a d_{x}^{\mathscr{A}}(a)=a x-x a \quad(a \in \mathscr{A}) .
$$

It is easily seen that $a d_{x}^{\mathscr{A}}$ is a derivation. Derivation of this form is called inner derivation.

Let $\mathscr{A}$ be a Banach algebra and $X$ a Banach $\mathscr{A}$-bimodule. Then $X^{*}$, the dual space of $X$, is a Banach $\mathscr{A}$-bimodule for operations given by

$$
\begin{gathered}
\langle x, a \xi\rangle=\langle x a, \xi\rangle, \quad\langle x, \xi a\rangle=\langle a x, \xi\rangle \\
\left(a \in \mathscr{A}, x \in X, \xi \in X^{*}\right) .
\end{gathered}
$$

$X^{*}$ is the dual module of $X$; and in particular $\mathscr{A}^{*}$ is the dual module of $\mathscr{A}$.

A Banach algebra $\mathscr{A}$ is called amenable if, for any $\mathscr{A}$ bimodule $X$, any derivation $D: \mathscr{A} \rightarrow X^{*}$ is inner. This definition of amenability was introduced by Johnson in [3]. A Banach algebra $\mathscr{A}$ is called weakly amenable if any derivation $D: \mathscr{A} \rightarrow \mathscr{A}^{*}$ is inner. Trivially, any amenable Banach algebra is weakly amenable.

Let $G$ be a locally compact group; a mean on $L^{\infty}(G)$ is a positive functional $m \in L^{\infty}(G)^{*}$ such that

$$
\langle 1, m\rangle=\|m\|=1 .
$$

A mean $m$ on $L^{\infty}(G)$ is called left invariant if

$$
\left\langle\delta_{x} * \phi, m\right\rangle=\langle\phi, m\rangle \quad\left(x \in G, \phi \in L^{\infty}(G)\right) .
$$

A locally compact group $G$ is called amenable if there exists a left invariant mean on $L^{\infty}(G)$. Note that $G$ is amenable if and only if $L^{1}(G)$ is an amenable Banach algebra.

A derivation $D: \mathscr{A} \rightarrow X$ is called approximately inner if there exists net $\left(\xi_{a}\right) \subseteq X$ such that, for every $a \in \mathscr{A}$,

$$
D(a)=\operatorname{norm}-\lim _{a}\left(a \cdot \xi_{a}-\xi_{a} \cdot a\right) .
$$

Recall from [1] that a Banach algebra $\mathscr{A}$ is called approximately amenable if, for any $\mathscr{A}$-bimodule $X$, any derivation $D: \mathscr{A} \rightarrow X^{*}$ is approximately inner.
2.2. Banach Spaces. For $n \in \mathbb{N}=\{1,2, \ldots\}$, we set $\mathbb{N}_{n}=$ $\{1, \ldots, n\}$. Let $E$ be a normed space. For each $k \in \mathbb{N}$, we denote by $E^{k}$ the linear space direct product of $k$ copies of $E$. The closed unit ball of $E$ is denoted by $B_{1}(E)$. We denote the dual of $E$ by $E^{*}$; the action of $\lambda \in E^{*}$ on an element $x \in E$ is written as $\langle x, \lambda\rangle$.

Following the notation of $[4,5]$ we define the weak $p$ summing norm (for $1 \leq p<\infty$ ) on $E^{n}$ by

$$
\mu_{p, n}(x)=\sup \left\{\left(\sum_{i=1}^{n}\left|\left\langle x_{i}, \lambda\right\rangle\right|^{p}\right)^{1 / p}: \lambda \in B_{1}\left(E^{*}\right)\right\},
$$

where $x=\left(x_{1}, \ldots, x_{n}\right) \in E^{n}$. See also [6,7]. Notice that, by the weak ${ }^{*}$ density of $B_{1}(E)$ in $B_{1}\left(E^{* *}\right)$, the weak $p$-summing norm on $\left(E^{*}\right)^{n}$ can also be computed as

$$
\mu_{p, n}(\lambda)=\sup \left\{\left(\sum_{i=1}^{n}\left|\left\langle x, \lambda_{i}\right\rangle\right|^{p}\right)^{1 / p}: x \in B_{1}(E)\right\},
$$

where $\lambda=\left(\lambda_{1}, \ldots, \lambda_{n}\right) \in\left(E^{*}\right)^{n}$.

2.3. Multinormed Spaces. The following definition is by Dales and Polyakov. For a full account of the theory of multinormed spaces, see [4].

Definition 1. Let $(E,\|\cdot\|)$ be a normed space, and let $\left(\|\cdot\|_{n}\right.$ : $n \in \mathbb{N}$ ) be a sequence such that $\|\cdot\|_{n}$ is a norm on $E^{n}$ for each $n \in \mathbb{N}$, with $\|\cdot\|_{1}=\|\cdot\|$ on $E$. Then the sequence $\left(\|\cdot\|_{n}\right.$ : $n \in \mathbb{N}$ ) is a multinorm if the following axioms hold (where in each case the axiom is required to hold for all $n \geq 2$ and all $\left.x_{1}, \ldots, x_{n} \in E\right)$ :

(A1) $\left\|x_{\sigma(1)}, \ldots, x_{\sigma(n)}\right\|_{n}=\left\|\left(x_{1}, \ldots, x_{n}\right)\right\|_{n}$ for each permutation $\sigma$ of $\mathbb{N}_{n}$

(A2) $\left\|\left(\alpha_{1} x_{1}, \ldots, \alpha_{n} x_{n}\right)\right\|_{n} \leq \max _{i \in \mathbb{N}_{n}}\left|\alpha_{i}\right|\left\|\left(x_{1}, \ldots, x_{n}\right)\right\|_{n}\left(\alpha_{1}\right.$, $\left.\ldots, \alpha_{n}\right) \in \mathbb{C}$

(A3) $\left\|\left(x_{1}, \ldots, x_{n-1}, 0\right)\right\|_{n}=\left\|\left(x_{1}, \ldots, x_{n-1}\right)\right\|_{n-1}$;

(A4) $\left\|\left(x_{1}, \ldots, x_{n-2}, x_{n-1}\right)\right\|_{n}=\left\|\left(x_{1}, \ldots, x_{n-2}, x_{n-1}\right)\right\|_{n-1}$.

The normed space $E$ equipped with a multinorm is a multinormed space, denoted in full by $\left(\left(E^{n},\|\cdot\|_{n}\right): n \in \mathbb{N}\right)$. We say that such a multinorm is based on $E$.

Definition 2. Let $\left(\left(E^{n},\|\cdot\|_{n}: n \in \mathbb{N}\right)\right.$ be a multinormed space. A subset $B \subset E$ is multibounded if

$$
\operatorname{mb}(B):=\sup \left\{\left\|\left(x_{1}, \ldots, x_{n}\right)\right\|_{n}: x_{1}, \ldots, x_{n} \in B, n \in \mathbb{N}\right\}<\infty .
$$

The constant $\mathrm{mb}(B)$ is the multibound of $B$.

The following easy remark is from [4, Proposition 6.5(ii)].

Remark 3. Let $E$ be a multinormed space. Then the absolutely convex hull of a multibounded set is multibounded, with the same multibound. 
2.4. The $(p, q)$-Multinorm. Following [4], we now introduce an important class of multinorms. Let $E$ be a normed space, and take $p, q$ with $1 \leq p, q<\infty$. For each $n \in \mathbb{N}$ and each $x=\left(x_{1}, \ldots, x_{n}\right) \in E^{n}$, we define

$$
\begin{aligned}
& \|x\|_{n}^{(p, q)} \\
& =\sup \left\{\left(\sum_{i=1}^{n}\left|\left\langle x_{i}, \lambda_{i}\right\rangle\right|^{q}\right)^{1 / q}:\right. \\
& \left.\lambda=\left(\lambda_{1}, \ldots, \lambda_{n}\right) \in\left(E^{*}\right)^{n}, \mu_{p, n}(\lambda) \leq 1\right\} .
\end{aligned}
$$

It is clear that $\|\cdot\|_{n}^{(p, q)}$ is a norm on $E^{n}$. As proved in [4], in the case where $1 \leq p \leq q<\infty$, the sequence $\left(\|\cdot\|_{n}^{(p, q)}: n \in \mathbb{N}\right)$ is a multinorm based on $E$.

Definition 4. Let $E$ be a normed space, and take $p, q$ with $1 \leq$ $p \leq q<\infty$. Then the multinorm $\left(\|\cdot\|_{n}^{(p, q)}: n \in \mathbb{N}\right)$ described above is the $(p, q)$-multinorm over $E$.

A subset of $E$ is $(p, q)$-multibounded if it is multibounded with respect to the $(p, q)$-multinorm. The $(p, q)$-multibound of such a set $B$ is denoted by $\mathrm{mb}_{p, q}(B)$.

Lemma 5. Let $E$ be a normed space, and take $p, q$ with $1 \leq p$, $q<\infty$. Then, for each $n \in \mathbb{N}$ and $\lambda=\left(\lambda_{1}, \ldots, \lambda_{n}\right) \in\left(E^{*}\right)^{n}$, we have

$$
\begin{aligned}
& \|\lambda\|_{n}^{(p, q)} \\
& =\sup \left\{\left(\sum_{i=1}^{n}\left|\left\langle x_{i}, \lambda_{i}\right\rangle\right|^{q}\right)^{1 / q}:\right. \\
& \left.x=\left(x_{1}, \ldots, x_{n}\right) \in\left(E^{*}\right)^{n}, \mu_{p, n}(x) \leq 1\right\} .
\end{aligned}
$$

Proof. This is proved in [4, Proposition 4.10]; it follows from the Principal of Local Reflexivity.

\subsection{Left ( $p, q)$-Multiinvariant Means}

Definition 6. Let $G$ be a locally compact group, and take $p, q$ with $1 \leq p \leq q<\infty$. A functional $\Lambda \in L^{\infty}(G)^{*}$ is left $(p, q)$ multiinvariant if the set $\{s \cdot \Lambda: s \in G\}$ is multibounded in the $(p, q)$-multinorm. The group $G$ is left $(p, q)$-amenable if there exists a left $(p, q)$-multiinvariant mean on $L^{\infty}(G)$.

The idea behind this definition is to attempt to measure the "left invariance" of a mean $\Lambda \in L^{\infty}(G)^{*}$ by measuring the growth of the sets $\{s \cdot \Lambda: s \in F\}$ as $F$ ranges through all the finite subsets of $G$. See [2] for more details.

\section{Multinorms and Approximate Amenability of Weighted Group Algebras}

Ghahramani and Loy in [1] proved that for a locally compact group $G$ the Banach algebra $L^{1}(G)$ is approximately amenable if and only if $G$ is amenable. In this section we prove that, if $\omega \geq 1$ is any weight function on $G$ and $L^{1}(G, \omega)$ is approximately amenable, then $G$ is left $(p, q)$-amenable. Through an example we show is not valid in general.

Theorem 7. Let $G$ be a locally compact group, and let $\omega$ be a weight function on $G$ such that $\omega(x) \geq 1(x \in G)$. If $L^{1}(G, \omega)$ is approximately amenable, then $G$ is left $(p, q)$-amenable.

Proof. By the following operations, $L^{\infty}\left(G, \omega^{-1}\right)$ is a Banach $M(G, \omega)$-bimodule;

$$
\langle f, \phi \cdot \mu\rangle=\langle\mu * f, \phi\rangle, \quad \mu \cdot \phi=\mu(G) \phi,
$$

where $\mu \in M(G, \omega), \phi \in L^{\infty}\left(G, \omega^{-1}\right)$, and $f \in L^{1}(G, \omega)$. Note that we have

$$
(\mu * f)(x)=\int_{G} f\left(y^{-1} x\right) d \mu(y)
$$

for $\mu \in M(G, \omega), f \in L^{1}(G, \omega)$, and $x \in G$. For every $\mu \in$ $M(G, \omega)$ and $f \in L^{\infty}\left(G, \omega^{-1}\right)$,

$$
\begin{aligned}
\langle f, 1 \cdot \mu\rangle & =\langle\mu * f, 1\rangle=\int(\mu * f)(x) d x \\
& =\iint f\left(y^{-1} x\right) d \mu(y) d x \\
& =\iint f(x) d x d \mu(y) \\
& =\mu(G) \int f(x) d x \\
& =\mu(G)\langle f, 1\rangle .
\end{aligned}
$$

Thus $1 \cdot \mu=\mu(G) \cdot 1 \in \mathbb{C} 1$; and by definition we have $\mu \cdot 1 \in \mathbb{C} 1$; that is, the space $\mathbb{C} 1$ is a submodule of $M(G, \omega)$. If we set $Z=$ $L^{\infty}\left(G, \omega^{-1}\right) / \mathbb{C} 1$, then $Z$ is a $M(G, \omega)$-bimodule, with $Z^{*}=$ $\left\{m \in L^{\infty}\left(G, \omega^{-1}\right)^{*} \mid m(1)=0\right\}$. By Hahn Banach Theorem there is $v \in L^{\infty}\left(G, \omega^{-1}\right)^{*}$ such that $v(1)=1$. It is easy to prove that the mapping $\widetilde{D}: M(G, \omega) \rightarrow Z^{*}$, defined by

$$
\begin{gathered}
\widetilde{D}(\mu)(\phi+\mathbb{C} 1)=(\mu \cdot v-\mu(G) v)(\phi) \\
\left(\mu \in M(G, \omega), \phi \in L^{\infty}\left(G, \omega^{-1}\right)\right),
\end{gathered}
$$

is a well-defined derivation. If the restrict ion $\widetilde{D}$ to $L^{1}(G, \omega)$ is denoted by $D$, then from the approximate amenability of $L^{1}(G, \omega)$ it follows that there is a net $\left(m_{i}\right)$ in $Z^{*}$ such that for every $\mu \in L^{1}(G, \omega)$ we have

$$
D(\mu)=\mu \cdot v-v \mu(G)=\lim _{i}\left(\mu \cdot m_{i}-m_{i} \mu(G)\right) .
$$

For $\mu \in L^{1}(G, \omega)$ with $\mu(G)=1$ and $\phi \in L^{\infty}\left(G, \omega^{-1}\right), \mu \phi=\phi$. Hence for every $x \in G, \phi \in L^{\infty}\left(G, \omega^{-1}\right)$, we have

$$
\begin{aligned}
\left\langle\phi+\mathbb{C} 1, D\left(\delta_{x}\right) \cdot \mu\right\rangle & =\left\langle\mu(\phi+\mathbb{C} 1), D\left(\delta_{x}\right)\right\rangle \\
& =\left\langle\mu \cdot \phi+\mathbb{C} 1, D\left(\delta_{x}\right)\right\rangle \\
& =\left\langle\phi+\mathbb{C} 1, D\left(\delta_{x}\right)\right\rangle .
\end{aligned}
$$


Thus $D\left(\delta_{x}\right) \cdot \mu=D\left(\delta_{x}\right)$. Therefore for every $x \in G$ and $\mu \epsilon$ $L^{1}(G, \omega)$ with $\mu(G)=1$ we have

$$
\begin{aligned}
\delta_{x} \cdot v-v=D\left(\delta_{x}\right)=D\left(\delta_{x}\right) \cdot \mu \\
=D\left(\delta_{x} * \mu\right)-\delta_{x} D(\mu) \\
=\lim _{i}\left[\left(\delta_{x} * \mu\right) \cdot m_{i}-m_{i}\left(\delta_{x} * \mu\right)\right. \\
\left.\quad-\delta_{x}\left(\mu \cdot m_{i}-m_{i} \cdot \mu\right)\right] \\
=\lim _{i}\left[\left(\delta_{x} * \mu\right) \cdot m_{i}\right. \\
\left.\quad-m_{i}-\delta_{x}\left(\mu \cdot m_{i}-m_{i}\right)\right] \\
=\lim _{i}\left(\delta_{x} m_{i}-m_{i}\right) .
\end{aligned}
$$

Thus

$$
\lim _{i} \delta_{x}\left(v-m_{i}\right)-\left(v-m_{i}\right)=0 .
$$

Since $\left\langle v-m_{i}, 1\right\rangle=1$, it follows that $\lim _{i}\left\|v-m_{i}\right\| \neq 0$, so there exists a subnet $\left(m_{j}\right)$ of $\left(m_{i}\right)$ such that $\left\|v-\widetilde{m}_{j}\right\| \neq 0$. For every $j$, if we set

$$
\widetilde{k}_{j}=\frac{v-\widetilde{m}_{j}}{\left\|v-\widetilde{m}_{j}\right\|},
$$

then $\widetilde{k}_{j} \in L^{\infty}\left(G, \omega^{-1}\right)$. If for each $j$ the restriction of $\widetilde{k}_{j}$ to $L^{\infty}(G)$ is denoted by (26) we have norm- $\lim _{j}\left(\delta_{x} \cdot k_{j}-k_{j}\right)=0$, for every $x \in G$. Since the space $L^{\infty}(G)$ is a commutative $C^{*}$ algebra with identity 1 , there is a compact space $T$ such that $L^{\infty}(G)^{*}=M(T)$. Since, for each $x \in G$ and $m \in M(T)$, we have $\left|\delta_{x} \cdot m\right|=\delta_{x}|m|$, therefore

$$
\begin{aligned}
\left|\delta_{x} \cdot\right| k_{j}|-| k_{j}|| & =\| \delta_{x} \cdot k_{j}|-| k_{j}|| \\
& \leq\left|\delta_{x} \cdot k_{j}-k_{j}\right| \quad(x \in G),
\end{aligned}
$$

and since $\lim _{j}\left|\delta_{x} \cdot k_{j}-k_{j}\right|=0$, thus, for each $x \in G$,

$$
\operatorname{norm}-\lim _{j}\left(\delta_{x} \cdot\left|k_{j}\right|-\left|k_{j}\right|\right)=0 \text {. }
$$

Let $k$ be a weak ${ }^{*}$-cluster point of $\left(k_{j}\right)$. Clearly $k$ is positive; take $p, q$ with $1 \leq p \leq q<\infty$. Recall that $L^{\infty}(G)^{*}$ can be identified isometrically as a Banach lattice with $L^{1}(\Omega)$ for some measure space $(\Omega, \mu)$. Set $\widehat{k}=|k| /\|k\|$. Since $\||k|\|=$ $\|k\|=\langle 1,|k|\rangle$, it is clear that $\widehat{k}$ is a mean on $L^{\infty}(G)$. Since $\mu_{p, n}\left(\varphi_{1}, \ldots, \varphi_{n}\right)=\mu_{p, n}\left(\psi_{1}, \ldots, \psi_{n}\right)$ for every $n \in \mathbb{N}$ and every $\varphi_{1}, \ldots, \varphi_{n}, \psi_{1}, \ldots, \psi_{n} \in L^{\infty}(\Omega)$ with $\left|\varphi_{i}\right|=\left|\psi_{i}\right|\left(i \in \mathbb{N}_{n}\right)[5$, $2.6]$, we see that

$$
\begin{array}{r}
\left\|\left(k_{1}, \ldots, k_{n}\right)\right\|_{n}^{(p, q)}=\left\|\left(\left|k_{1}\right|, \ldots,\left|k_{n}\right|\right)\right\|_{n}^{(p, q)} \\
\left(k_{1}, \ldots, k_{n} \in L^{\infty}(G)^{*}\right) .
\end{array}
$$

Now note that $|s \cdot k|=s \cdot|k|$ for every $s \in G$, and so $\{s \cdot \widehat{k}: s \in G\}$ is multibounded in the $(p, q)$-multinorm. The result follows, and $G$ is left $(p, q)$-amenable.
Lemma 8. Let $\mathscr{A}$ be a commutative Banach algebra. If $\mathscr{A}$ is approximately amenable, then $\mathscr{A}$ is weakly amenable.

Proof. Suppose that $D: \mathscr{A} \rightarrow \mathscr{A}^{*}$ is a derivation we show that $D$ is inner. Since $\mathscr{A}$ is approximately amenable and commutative, so there exists a net $\left(\xi_{i}\right)$ in $\mathscr{A}^{*}$ such that for each $a \in \mathscr{A}$

$$
D(a)=\operatorname{norm}-\lim _{i}\left(a \cdot \xi_{i}-\xi_{i} \cdot a\right)=0 .
$$

On the other hand since $\mathscr{A}$ is commutative, every inner derivation on $\mathscr{A}$ is zero, so $D$ is inner. Therefore $\mathscr{A}$ is weakly amenable.

The Example 6.2 of [1] shows that an approximately amenable Banach algebra need not be weakly amenable.

The following example shows that the opposite of Theorem 7 is not true in general.

Example 9. If we define the weight function $\omega$ on $(\mathbb{Z},+)$ by $\omega(n)=1+|n|$ for every $n \in \mathbb{Z}$, then the Banach algebra $l^{1}(\mathbb{Z}, \omega)$ is not approximately amenable.

Proof. Let

$$
l^{1}(\mathbb{Z}, \omega)=\left\{a=(a(n): n \in \mathbb{Z})\left|\sum_{-\infty}^{\infty}\right| a(n) \mid \omega(n) \leq \infty\right\} .
$$

Then $l^{1}(\mathbb{Z}, \omega)$ is a commutative Banach algebra with respect to convolution multiplication,

$$
\begin{array}{r}
(a * b)(n)=\sum_{k=-\infty}^{\infty} a(n-k) b(k) \\
\left(n \in \mathbb{Z}, a, b \in l^{1}(\mathbb{Z}, \omega)\right),
\end{array}
$$

and the norm

$$
\|a\|=\sum_{-\infty}^{\infty}|a(n)| \omega(n)<\infty \quad\left(a \in l^{1}(\mathbb{Z}, \omega)\right) .
$$

We show that $l^{1}(\mathbb{Z}, \omega)$ is not approximately amenable. To see this note that

$$
\begin{gathered}
\sup \left\{\frac{\omega(n+m)}{\omega(n) \omega(m)} \cdot \frac{1+|n|}{1+|n+m|} \mid n, m \in \mathbb{Z}\right\} \\
=\sup \left\{\frac{1}{1+|m|} \mid m \in \mathbb{Z}\right\}=1 .
\end{gathered}
$$

So by Theorem 2.3 of $[8], l^{1}(\mathbb{Z}, \omega)$ is not weakly amenable. On the other hand, since $l^{1}(\mathbb{Z}, \omega)$ is a commutative Banach algebra and if it is approximately amenable, so by Lemma 8 it is weakly amenable, and this is a contradiction. So $l^{1}(\mathbb{Z}, \omega)$ is not approximately amenable.

Let $\omega$ be a weight function on $G$; for $x \in G$, we define $\omega^{*}(x)=\omega(x) \omega\left(x^{-1}\right)$. In the following theorem we prove that if $\omega^{*}$ is bounded on $G$ then the opposite of Theorem 7 is true. 
Theorem 10. Suppose that $\omega$ is a weight function on a locally compact group $G$ such that $\omega \geq 1$ and $\omega^{*}$ is bounded. Then $G$ is left $(p, q)$-amenable if and only if $L^{1}(G, \omega)$ is approximately amenable.

Proof. $G$ is left $(p, q)$-amenable, so suppose that $\Lambda$ is a left $(p, q)$-invariant mean on $L^{\infty}(G)$; that is, $\{s \cdot \Lambda: s \in G\}$ is $(p, q)$-multibounded on $\mathrm{L}^{\infty}(G)^{*}$. By Remark 3 or the KreinSmulian theorem, the closed convex hull $K$ of $\{s \cdot \Lambda: s \in G\}$ is weakly compact. For each $s \in G$, consider that the map $L_{s}: K \rightarrow K$ defined by

$$
L_{s}(\varphi)=s \cdot \varphi \quad(s \in G, \varphi \in K) .
$$

We obtain a group $\Sigma:=\left\{L_{s}: s \in G\right\}$ of isometric affine maps. By Ryll-Nardzewski fixed point theorem, given in $[9,10]$, there exists $\Lambda_{0} \in K$ which is a common fixed point for the set $\left\{L_{s}: s \in G\right\}$. Obviously, $\Lambda_{0}$ must be a left-invariant mean on $L^{\infty}(G)$. Hence the group $G$ is amenable and $\omega^{*}$ is bounded on $G$; by Theorem 0 of [11] $L^{1}(G, \omega)$ is amenable, so $L^{1}(G, \omega)$ is approximately amenable.

\section{Approximate Amenability $M(G, \omega)$ of a Locally Compact Group $G$}

It is standard that $L^{1}(G)$ always has a bounded approximate identity which is a net consisting of continuous functions of compact support, and this net is clearly also a bounded approximate identity for $L^{1}(G, \omega)$.

Let $\omega$ be a weight function on $G$ with $\omega(x) \geq 1(x \in G)$; then, with the convolution product $*$ given by

$$
\begin{gathered}
\langle\phi, \mu * v\rangle=\iint_{G} \phi(s t) d \mu(s) d \nu(t) \\
\left(\mu, \nu \in M(G, \Omega), \phi \in C_{0}(G, \omega)\right),
\end{gathered}
$$

the Banach space $M(G, \omega)$ defines a unital convolution Banach algebra for which $L^{1}(G, \omega)$ is a closed ideal.

In the following lemma we show that if $M(G, \omega)$ is approximately amenable then $G$ is left $(p, q)$-amenable.

Theorem 11. Let $G$ be a locally compact group. If $M(G, \omega)$ is approximately amenable then $G$ is left $(p, q)$-amenable.

Proof. Since $L^{1}(G, \omega)$ is a closed two sided ideal of $M(G, \omega)$ and has an approximate identity, from Corollary 2.3 of [1] it follows that $L^{1}(G, \omega)$ is approximately amenable. So by Theorem 7, $G$ is left $(p, q)$-amenable.

It is well known (c.f. [12]) that if $G$ is amenable and $\omega^{*}$ is bounded on $G$, then there is a continuous positive character $\phi$ on $G$ such that

$$
\phi \leq \omega \leq c \phi \quad \text { such that } c=\sup \left\{\omega^{*}(x) \mid x \in G\right\} .
$$

In particular,

$$
\begin{aligned}
& L^{1}(G, \omega)=L^{1}(G, \phi) \simeq L^{1}(G), \\
& M(G, \omega)=M(G, \phi) \simeq M(G) .
\end{aligned}
$$

Theorem 12. If $M(G, \omega)$ is approximately amenable and $\omega^{*}$ is bounded on $G$, then $G$ is a discrete group.

Proof. Since $M(G, \omega)$ is approximately amenable, from Theorem 11 we conclude that $G$ is left $(p, q)$-amenable. Using the fact that $\omega^{*}$ is bounded, we infer that $M(G, \omega) \simeq M(G)$. So by Theorem 1.1 of [13] $G$ is discrete.

\section{Conflict of Interests}

The author declares that there is no conflict of interests regarding the publication of this paper.

\section{Acknowledgments}

This research was done while the author was at the Department of Pure Mathematics, Isfahan University of Technology, during the years 2012-2013. The author would like to express his thanks to Professor Rasoul Nasr-Isfahani and Professor Mehdi Nemati in the Banach algebras and Operator Theory for warm hospitality and great scientific atmosphere. Last but not least, He thanks his family for all their support.

\section{References}

[1] F. Ghahramani and R. J. Loy, "Generalized notions of amenability," Journal of Functional Analysis, vol. 208, no. 1, pp. 229-260, 2004.

[2] H. G. Dales, M. Daws, H. L. Pham, and P. Ramsden, "Multinorms and the injectivity of $L^{p}(G)$," London Mathematical Society, vol. 86, no. 3, pp. 779-809, 2012.

[3] B. E. Johnson, Cohomology in Banach Algebra, vol. 127 of Memoirs of the American Mathematical Society, 1972.

[4] H. G. Dales and M. E. Polyakov, "Multi-normedspaces," Dissertationes Mathematicae (Rozprawy Matematyczne). In press, http://arxiv.org/abs/1112.5148.

[5] G. J. O. Jameson, Summing and Nuclear Norms in Banach Space Theory, vol. 8 of London Mathematical Society Student Texts, Cambridge University Press, 1987.

[6] J. Diestel, H. Jarchow, and A. Tonge, Absolutely Summing Operators, vol. 43 of Cambridge Studies in Advanced Mathematics, Cambridge University Press, 2000.

[7] R. Ryan, Introduction to Tensor Products of Banach Spaces, Springer Monographs in Mathematics, Springer, London, UK, 2012.

[8] W. G. Bade, P. C. Curtis Jr., and H. G. Dales, "Amenability and weak amenability for Beurling and Lipschitz-algebras," Proceedings of the London Mathematical Society, vol. 55, pp. 359377, 1987.

[9] F. P. Greeleaf, Invariant Means on Topological Groups, Van Nostrand Mathematical Studies, vol. 16, van Nostrand Reinhold, New York, NY, USA, 1969.

[10] A. L. T. Paterson, Amenability, Mathematical Surveys and Monographs, vol. 29, American Mathematical Society, Providence, RI, USA, 1988.

[11] N. Gronback, "Amenability of weighted convolution algebras on locally compact groups," Transactions of the American Mathematical Society, vol. 319, pp. 765-779, 1990.

[12] M. C. White, "Characters on weighted amenable groups," Bulletin London Mathematical Society, vol. 23, pp. 375-380, 1991. 
[13] H. G. Dales, F. Ghahramani, and A. Y. Helemskii, "The amenability of measure algebras," Journal of the London Mathematical Society, vol. 66, no. 1, pp. 213-226, 2002. 


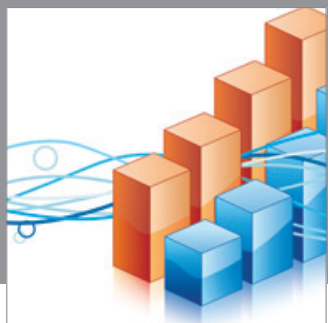

Advances in

Operations Research

mansans

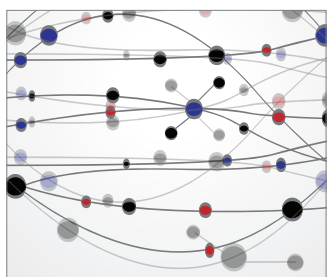

The Scientific World Journal
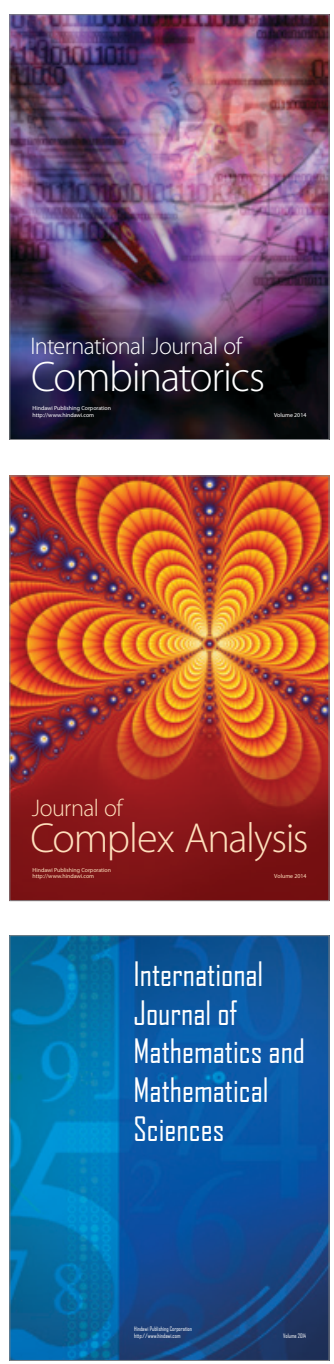
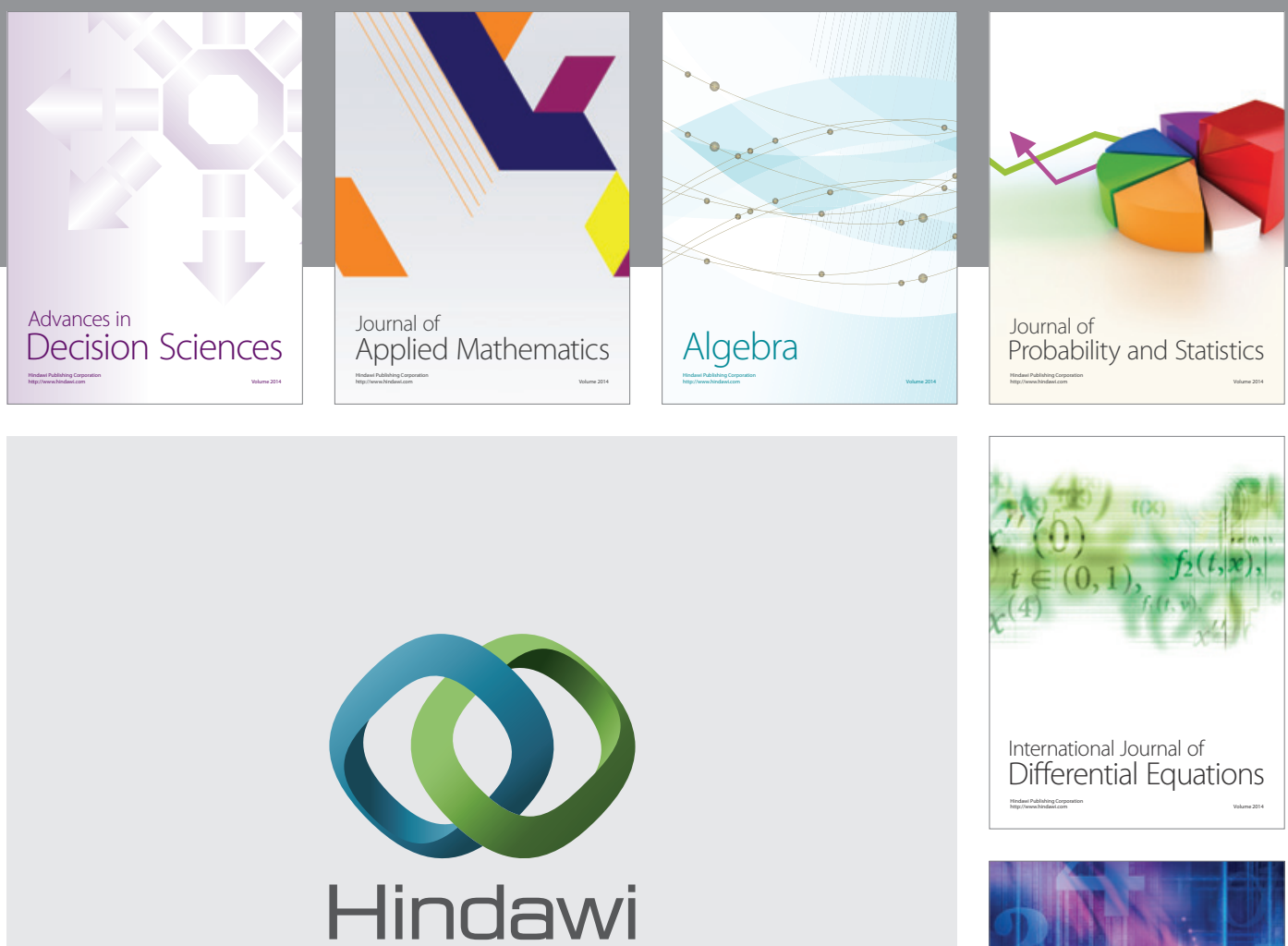

Submit your manuscripts at http://www.hindawi.com
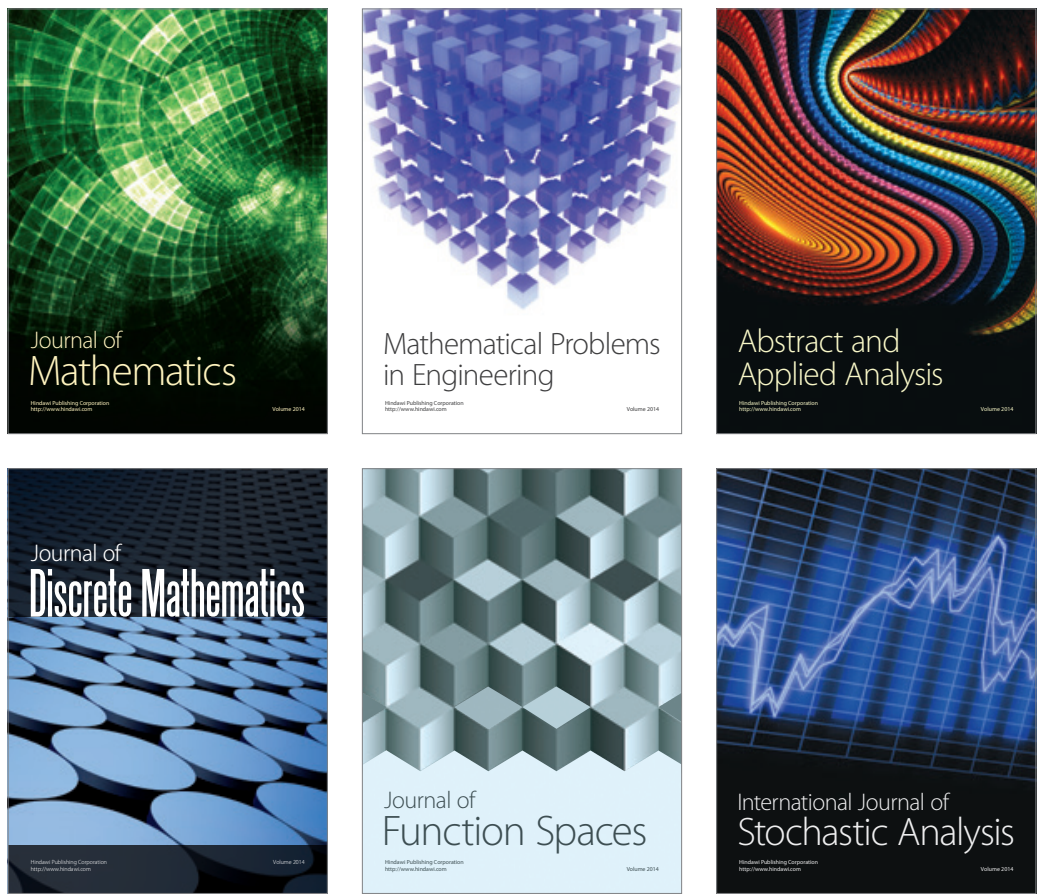

Journal of

Function Spaces

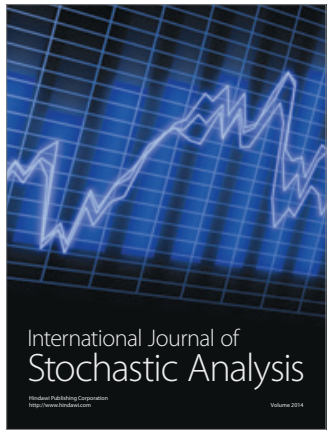

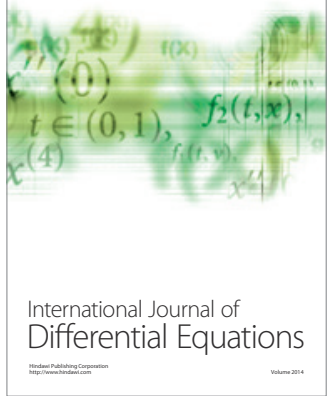
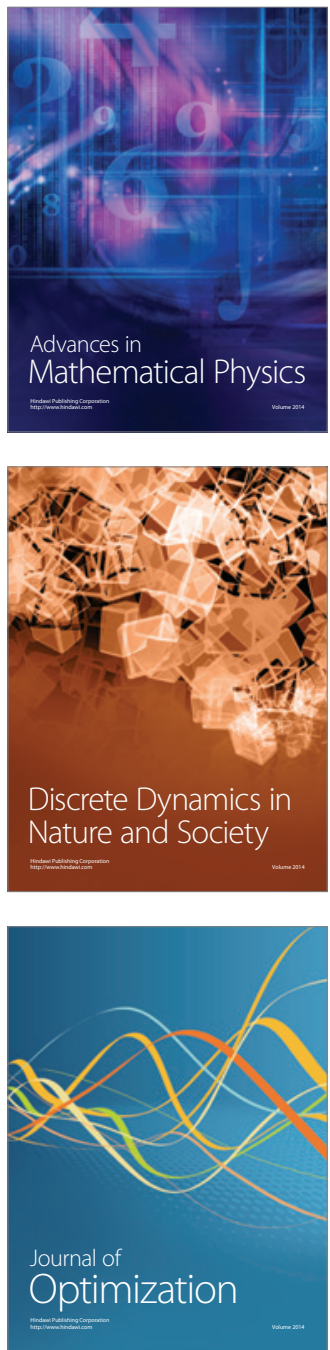